\title{
Antiferromagnetic Heisenberg model on an anisotropic triangular lattice in the presence of a magnetic field
}

\author{
Shun-Qing Shen ${ }^{1}$ and F. C. Zhang ${ }^{2}$ \\ ${ }^{1}$ Department of Physics, the University of Hong Kong, Pokfulam, Hong Kong, China \\ ${ }^{2}$ Department of Physics, University of Cincinnati, Cincinnati, Ohio 45221
}

(Received 12 October 2001; revised manuscript received 14 August 2002; published 21 November 2002)

\begin{abstract}
We use Schwinger boson mean field theory to study the antiferromagnetic spin-1/2 Heisenberg model on an anisotropic triangular lattice in the presence of a uniform external magnetic field. We calculate the field dependence of the spin incommensurability in the ordered spin spiral phase, and compare the results to the recent experiments in $\mathrm{Cs}_{2} \mathrm{CuCl}_{4}$ by Coldea et al. [Phys. Rev. Lett. 86, 1335 (2001)].
\end{abstract}

DOI: 10.1103/PhysRevB.66.172407

PACS number(s): 75.10.-b

The ground states of two-dimensional (2D) Heisenberg models continue to be of great interest. ${ }^{1,2}$ In this paper, we study the antiferromagnetic (AF) Heisenberg model on an anisotropic triangular lattice in the presence of an external magnetic field along the $z$ axis:

$$
H_{S}=\frac{1}{2} \sum_{i, \delta} J_{\delta} \mathbf{S}_{i} \cdot \mathbf{S}_{i+\delta}-\mu B \sum_{i} \mathbf{S}_{i}^{z} .
$$

In the above equation, the summation runs over all the lattice sites $i$ and their neighboring sites $(i+\delta)$. We consider AF nearest neighbor spin-spin couplings, represented by $J$ and $J^{\prime}$ as shown in Fig. 1, with both $J$ and $J^{\prime} \geqslant 0$. In the absence of the field, this model is equivalent to a class of models recently considered by a number of authors. ${ }^{3-6}$ The model includes several well known limiting cases. At $J=0$, it is equivalent to a 2D square lattice model, whose ground state is a two-sublattice Néel phase. At $J^{\prime}=0$, it becomes a set of decoupled spin chains. At $J^{\prime}=J$, it is reduced to the isotropic triangular-lattice model, where the ground state is a three-sublattice antiferromagnet. Experimentally, this model may be relevant to the insulating phase of the layered molecular crystals, $\kappa$-(BEDT-TTF) ${ }_{2} X$ (Ref. 7) and $\theta$-(BEDT-TTF $)_{2} \operatorname{RbZn}(\mathrm{SCN})_{4} \cdot{ }^{8}$ Our interest in this model is largely motivated by recent experiments on $\mathrm{Cs}_{2} \mathrm{CuCl}_{4}$. That system is a quasi-2D $S=1 / 2$ frustrated Heisenberg antiferromagnet. ${ }^{9}$ Coldea et al. ${ }^{10}$ used neutron scattering to study the ground state and dynamics of the system in high magnetic fields. Among the observations, these authors found that the incommensurate wave vector changes as the magnetic field increases, and the spiral spin density wave evolves into a fully saturated state.

In this paper we apply the Schwinger boson mean field theory (MFT) to study the effect of a magnetic field in the frustrated Heisenberg models. This method enables us to study incommensurate magnetic ordering in quantum spin systems. The magnetic ordering is identified as the Bose condensation of the Schwinger bosons, and the incommensuration of the ordering is determined by the wave vector of the condensed Schwinger bosons. In the absence of a magnetic field, the MFT predicts three possible ground state: a twosublattice Neel phase, a spiral spin state, and a spin liquid phase, similar to the results obtained in the high temperature series expansions. ${ }^{3}$ In the presence of a field, we calculate the field dependence of the incommensuration in the spiral phase, and compare the results with the experimental observation in $\mathrm{Cs}_{2} \mathrm{CuCl}_{4}$, with good qualitative agreements.

In terms of Schwinger bosons $a_{i 1}$ and $a_{i 2}$, the spin operators are expressed as

$$
\mathbf{S}_{i}^{+}=a_{i 1}^{\dagger} a_{i 2} ; \mathbf{S}_{i}^{-}=a_{i 2}^{\dagger} a_{i 1} ; \mathbf{S}_{i}^{z}=\frac{1}{2}\left(a_{i 1}^{\dagger} a_{i 1}-a_{i 2}^{\dagger} a_{i 2}\right),
$$

with a local constraint at every site $i$ given by $a_{i 1}^{\dagger} a_{i 1}$ $+a_{i 2}^{\dagger} a_{i 2}=1$. As a standard method, we introduce a Lagrangian multiplier field $\lambda_{i}$ to describe the constraint. The Hamiltonian of the system then becomes

$$
\begin{aligned}
H= & -\frac{1}{8} \sum_{i, \delta, \mu, \nu} J_{\delta} A_{i j, \mu \nu}^{\dagger} A_{i j, \mu \nu}-\frac{h}{2}\left(a_{i 1}^{\dagger} a_{i 1}-a_{i 2}^{\dagger} a_{i 2}\right) \\
& +\sum_{i} \lambda_{i}\left(a_{i 1}^{\dagger} a_{i 1}+a_{i 2}^{\dagger} a_{i 2}-1\right)+\frac{1}{8} \sum_{i, \delta} J_{\delta},
\end{aligned}
$$

where $h=\mu B ; \quad \mu, \nu=1,2, \quad j=i+\delta$, and $A_{i j, \mu \nu}=a_{i \mu} a_{j \nu}$ $-a_{i \nu} a_{j \mu}$ is the spin singlet operator of bond $(i j)$. We note that operator $A_{i j, \mu \nu}$ is antisymmetric with respect to either the position $(i j)$ or the indices $(\mu \nu)$. On a bipartite lattice, a spin rotation by $\pi$ on one of the sublattices transforms the spin-singlet bond operators into a symmetric operator with respect to the bond indices $(i j)$. A mean field theory based

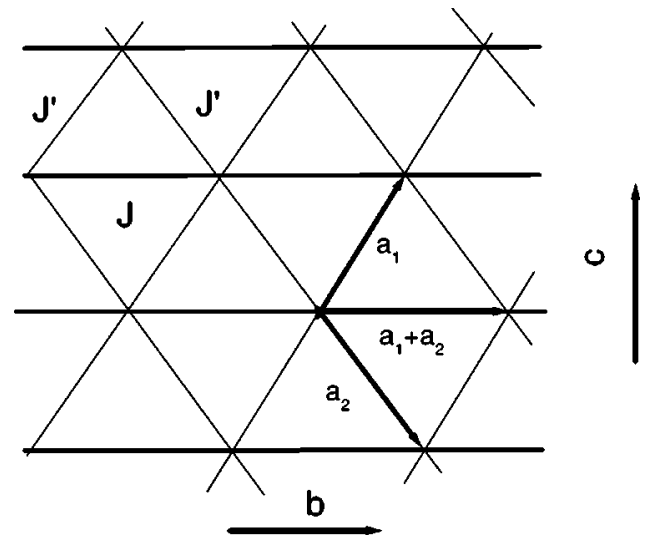

FIG. 1. The anisotropic triangle lattice: the bond along the $b$ axis is $J$ and the bonds along the $a_{1}$ and $a_{2}$ axes are $J^{\prime}$. 
on that transformation was developed by Auerbach and Arovas. ${ }^{11}$ The method was extended to study the frustrated lattices by many authors. ${ }^{12}$ (For an overview of Schwinger boson theory, see Ref. 2 and references therein.) We introduce two types of mean fields, $\Delta_{\mu \nu}(\delta) \equiv(1 / 2 i)\left\langle A_{j j+\delta, \mu \nu}\right\rangle$ and $\lambda=\left\langle\lambda_{j}\right\rangle$, where $\langle\cdots\rangle$ represents the thermodynamic average. The mean field Hamiltonian may be solved using the conventional bosonic Bogliubov transformation as well as the Green function's method.

The mean field Hamiltonian in Eq. (3) is diagonalized as

$$
H_{M F}=\sum_{\mathbf{k}, \mu= \pm} \omega_{\mu}(\mathbf{k})\left(\alpha_{\mathbf{k} \mu}^{\dagger} \alpha_{\mathbf{k} \mu}+\frac{1}{2}\right)+\mathcal{E}_{0},
$$

where the single boson spectra $\omega_{\mu}(\mathbf{k})=\omega(\mathbf{k}) \pm h / 2$ with $\omega(\mathbf{k})=\sqrt{\lambda^{2}-|\gamma(\mathbf{k})|^{2}}$ and $\gamma(\mathbf{k})=\Sigma_{\delta} J_{\delta} \Delta_{12}(\delta) \sin (\mathbf{k} \cdot \delta)$. $(\mu$ $= \pm) . \alpha_{\mathbf{k} \mu}$ is a boson annihilation operator related to the original Schwinger bosons. $\quad \mathcal{E}_{0} / N_{\Lambda}=\Sigma_{\delta, \mu \nu} J_{\delta}\left[\left|\Delta_{\mu \nu}(\delta)\right|^{2}\right.$ $+1 / 4] / 2-2 \lambda$. The free energy is given by $\left(\beta=1 / k_{B} T\right.$, with $T$ the temperature)

$F=\frac{1}{\beta} \sum_{\mathbf{k}, \mu= \pm} \ln \left[1-\exp \left[-\beta \omega_{\mu}(\mathbf{k})\right]+\frac{1}{2} \sum_{\mathbf{k}, \mu= \pm} \omega_{\mu}(\mathbf{k})+\mathcal{E}_{0}\right.$.

The mean field Hamiltonian is solved together with the selfconsistent equations for the two types of mean fields, which are given by

$$
\begin{gathered}
\frac{1}{N_{\Lambda}} \sum_{\mathbf{k}, \mu= \pm} \frac{\lambda}{\omega(\mathbf{k})}\left[n_{B}\left(\omega_{\mu}(\mathbf{k})\right)+1 / 2\right]=2, \\
\frac{1}{N_{\Lambda}} \sum_{\mathbf{k}, \mu= \pm} \frac{\gamma(\mathbf{k})}{\omega(\mathbf{k})} \sin (\mathbf{k} \cdot \delta)\left[n_{B}\left(\omega_{\mu}(\mathbf{k})\right)+1 / 2\right]=2 \Delta(\delta) .
\end{gathered}
$$

In the MFT, the magnetic ordering may be identified as the Schwinger boson condensation. ${ }^{13}$ Below we examine the possible Schwinger boson condensation at the wave vector $\mathbf{k}= \pm \mathbf{k}^{*}$, corresponding to the lowest energy of $\tilde{\omega}_{\mu}\left( \pm \mathbf{k}^{*}\right)$ $\rightarrow 0$. We introduce a non-negative quantity,

$$
b_{0}=\frac{2 \lambda}{N_{\Lambda} \omega_{-}\left(\mathbf{k}^{*}\right)}\left\{n_{B}\left[\omega_{-}\left(+\mathbf{k}^{*}\right)\right]+n_{B}\left[\omega_{-}\left(-\mathbf{k}^{*}\right)\right]\right\},
$$

such that the points $\mathbf{k}= \pm \mathbf{k}^{*}$ in the integrals in Eqs. (6a) and (6b) are taken into account separately:

$$
\begin{gathered}
\sum_{\mu= \pm} \int \frac{d \mathbf{k}^{\prime}}{(2 \pi)^{2}} \frac{\lambda}{\omega(\mathbf{k})}\left[n_{B}\left(\omega_{\mu}(\mathbf{k})\right)+1 / 2\right]=2-b_{0}, \\
\sum_{\mu= \pm} \int \frac{d \mathbf{k}^{\prime}}{(2 \pi)^{2}} \frac{\gamma(\mathbf{k})}{\omega(\mathbf{k})} \sin (\mathbf{k} \cdot \delta)\left[n_{B}\left(\omega_{\mu}(\mathbf{k})\right)+1 / 2\right] \\
=\frac{2 J_{\delta} \Delta(\delta)}{J_{\delta}}-b_{0} \gamma\left(\mathbf{k}^{*}\right) \sin \left(\mathbf{k}^{\star} \cdot \delta\right) / \lambda .
\end{gathered}
$$

The general features of the mean field solutions in 2D lattices are qualitatively given as below. At any finite $T, b_{0}$
$=0$. At $T=0$, if $\min [\omega(\mathbf{k})] \neq 0$ and $b_{0}=0$, the ground state is a spin liquid, whose gap depends on the minimal value of the spectra $\omega(\mathbf{k})$. If $\min [\omega(\mathbf{k})]=0$ and $b_{0}$ is finite, the Schwinger bosons are condensed to the lowest energy state, and the system possesses a magnetic long-range order. The ordering wave vector $Q$ is determined by $\omega\left(\mathbf{k}^{*}\right)=0$. From the spin-spin correlations,

$$
\chi_{\alpha \alpha}(\mathbf{q})=-\lim _{\tau \rightarrow 0^{-}}\left\langle T_{\tau} \mathbf{S}_{\mathbf{q}}^{\alpha}(\tau) \mathbf{S}_{-\mathbf{q}}^{\alpha}(0)\right\rangle(\alpha=x, y, z),
$$

we have

$$
\begin{aligned}
\chi_{x x}(\mathbf{q})= & \chi_{y y}(\mathbf{q})=\chi_{z z}(\mathbf{q})=\frac{1}{4 N_{\Lambda}} \sum_{\mathbf{k}}\left(\frac{n_{B}[\omega(\mathbf{k})]+\frac{1}{2}}{\omega(\mathbf{k})}\right) \\
& \times\left(\frac{n_{B}[\omega(\mathbf{k}+\mathbf{q})]+\frac{1}{2}}{\omega(\mathbf{k}+\mathbf{q})}\right)\left[\lambda^{2}-\gamma(\mathbf{k}) \gamma(\mathbf{k}+\mathbf{q})\right]-\frac{1}{16}
\end{aligned}
$$

for $h=0$, which indicates the mean field theory does not break $\mathrm{SU}(2)$ symmetry. In the thermodynamic limit $\left(N_{\Lambda} \rightarrow\right.$ $+\infty)$, the correlation functions are convergent except for $\mathbf{Q}$ $=2 \mathbf{k}^{*}$,

$$
\frac{\chi_{\alpha \alpha}(\mathbf{Q})}{N_{\Lambda}}=\frac{b_{0}^{2}}{8},
$$

which indicates that there exist long-range correlations with $\mathbf{Q}=2 \mathbf{k}^{*}$, from which $b_{0} \neq 0$.

On the triangular-lattice (see Fig. 1), each site has six neighbors: $\pm \mathbf{a}_{1}, \pm \mathbf{a}_{2}, \pm\left(\mathbf{a}_{1}+\mathbf{a}_{2}\right)$. It is convenient to write the wave vector $\mathbf{k}=\left(k_{1}, k_{2}\right)$, with $k_{1}$ and $k_{2}$ the components of the vector along the directions of $a_{1}$ and $a_{2}$, respectively. The lattice constant $a$ is set to 1 . The component of the vector along the $b$ axis is $k_{1}+k_{2}$. In the case the solution for $\mathbf{k}^{*}$ has the form $\mathbf{k}^{*}=\left(k_{0}^{*}, k_{0}^{*}\right)$. Thus the vector $\mathbf{k}^{*}$ is along the $b$ axis.

We now consider the mean field solutions at $T=0$. Let us first discuss the solutions in the absence of the field. At $J^{\prime} / J=1$ (the isotropic triangular lattice), $k_{0}^{*}=\pi / 3$, and $\omega\left(\mathbf{k}^{*}\right)=0, b_{0}$ is finite, indicating a magnetic long-range order with the ordering wave vector $\mathbf{Q}=(2 \pi / 3,2 \pi / 3)$. At $J$ $=0$ (the square lattice), $k_{0}^{*}=\pi / 2$ and $\omega\left(\mathbf{k}^{*}\right)=0$, and $b_{0}$ $>0$ implying a Neel ordering at $\mathbf{Q}=(\pi, \pi)$. The MFT in these two limiting cases is consistent with the known results. At $J^{\prime}=0$ (decoupled one-dimensional chains), the MFT gives $k_{0}^{*}=\pi / 4, \quad\left(2 k_{0}^{*}=\pi / 2\right.$ along the chains $), \omega\left(\mathbf{k}^{*}\right)>0$, and $b_{0}=0$, suggesting a spin gap state. The $1 \mathrm{D}$ model is exactly soluable, and the ground state is a gapless spin liquid, ${ }^{14}$ although the static sin-spin correlation becomes strongest at $Q_{b}=\pi .{ }^{15}$ The discrepancy between the MFT and the exact solutions is primarily due to the neglect of the topological term in the MFT. For the general values of $J^{\prime} / J$, the MFT predicts three phases at $h=0$ : (1) a spin liquid phase at $J^{\prime} / J<0.136$; (2) a spin spiral state at $0.136<J^{\prime} / J$ $<1.70$, with an ordering wave vector between $(\pi / 2, \pi / 2)$ 


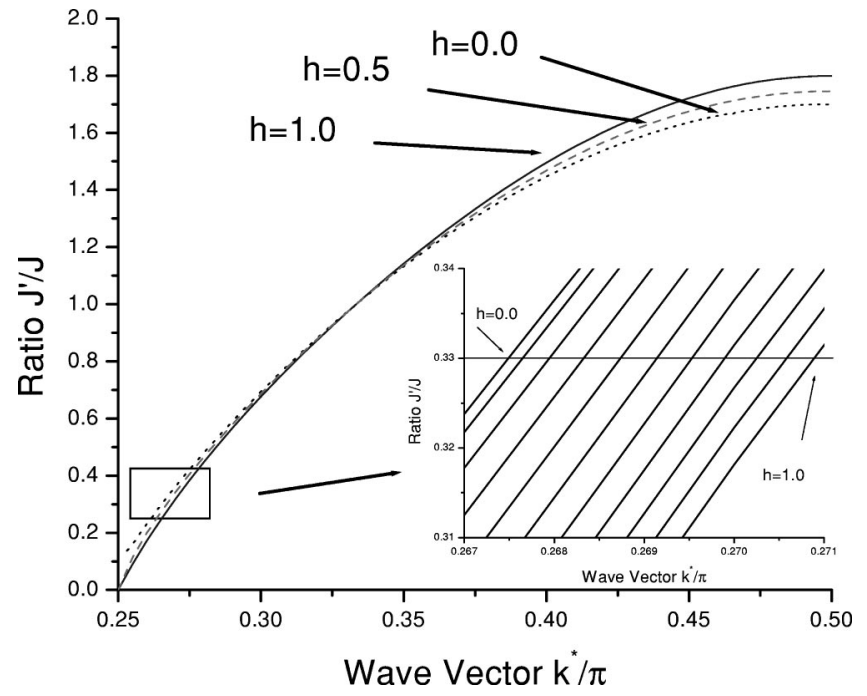

FIG. 2. The ratio vs the wave vector $k_{0}^{*}$ at different fields are plotted. Inset: the wave vector near $J^{\prime} / J=0.33$ at ten different fields are plotted. From left to right, $h^{*}=0.0,0.1, \ldots, 1.0$.

and $(\pi, \pi)$; and (3) an antiferromagnet with an ordering wave vector $\mathbf{Q}=(\pi, \pi)$ at $J^{\prime} / J>1.70$. We note that the phase diagram of the model was studied previously by using a series expansion technique, linear spin density wave and $\mathrm{SP}(N)$ mean field theory etc. ${ }^{3,5,6,12}$ In the method of series expansion, they found a Néel state persisting down to $J^{\prime} / J$ $>1.43$, and predicted a spiral phase at small ratio of $J^{\prime} / J$. These are consistent with our MFT. These authors also found a dimer phase between the Néel and spiral states. The dimer phase breaks translational invariance and is not included in the present MFT.

We now consider the solutions at $h \neq 0$. In this case, the $\mathrm{SU}(2)$ rotational symmetry is broken, and $\omega_{-}(\mathbf{k})<\omega_{+}(\mathbf{k})$. The Bose condensation criterion is given by $\omega_{-}\left(\mathbf{k}^{*}\right)$ $=\omega\left(\mathbf{k}^{*}\right)-h / 2=0$. We use the MFT to study the field dependent incommensurability in the spin spiral phase. The results are shown in Fig. 2, where the vector $\mathbf{k}^{*}$ are plotted as a function of $J^{\prime} / J$ at several values of $h$. The main feature is as follows. (1) $J^{\prime} / J=1$ is a stable fixed point, around which the external magnetic field does not change the ordering wave vector. (2) At $0.136<J^{\prime} / J<1$, the ordering wave vector increases slightly as the field increases. (3) At $J^{\prime} / J>1$, the ordering wave vector decreases as the field increases. (4) At $J^{\prime} / J<0.136$, the spin liquid may evolve into a spiral state, and becomes fully saturated as the field further increases.

In the presence of a magnetic field, the spin $z$ component $\left\langle S_{z}\right\rangle \neq 0$, and the ground state breaks the $\mathrm{SU}(2)$ invariance. At $T=0$, the expectation value of $S_{z}$ is given by

$$
\left\langle\mathbf{S}_{i}^{z}\right\rangle=\frac{1}{2} h^{*} b_{0}\left(\mathbf{k}^{*}, h^{*}\right)
$$

where $h^{*}=h /(2 \lambda)$ is the dimensionless field. At $h \neq 0,\left\langle\mathbf{S}_{i}^{z}\right\rangle$ is finite, which indicates a polarized component along the field orientation $z$ axis. The static transverse susceptibilities at $Q=2 k^{*}$ are given by

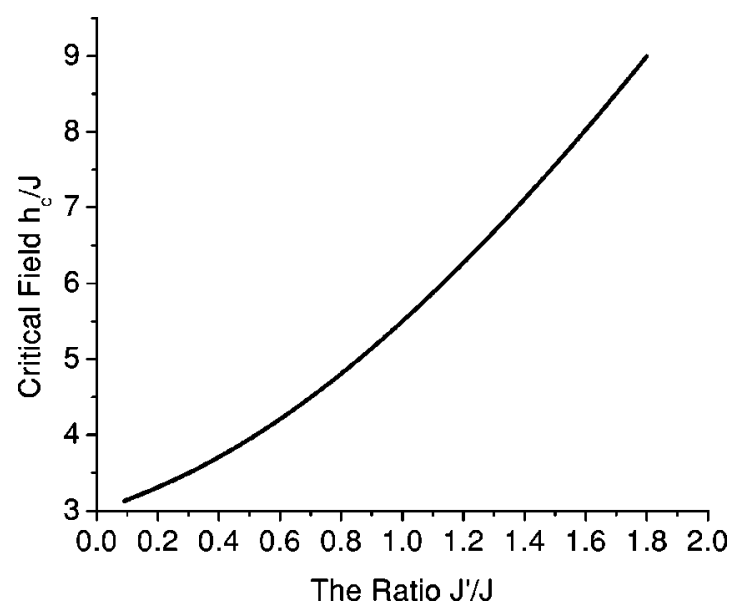

FIG. 3. The critical field in unit $J$ vs the ratio $J^{\prime} / J$.

$$
\frac{\chi_{\alpha \alpha}(\mathbf{Q})}{N_{\Lambda}}=\frac{b_{0}^{2}\left(\mathbf{k}^{*}, h^{*}\right)}{8}\left[1-\left(h^{*}\right)^{2}\right]
$$

for $\alpha=x$ and $y \cdot \chi_{\alpha \alpha}(\mathbf{Q}) / N_{\Lambda}$ decreases as the field increases, and approaches zero at $h^{*}=1 . b_{0}\left(\mathbf{k}^{*}, h^{*}\right)$ can be calculated within the MFT. A special case is $b_{0}\left(\mathbf{k}^{*}, h^{*}=1\right)=1$ corresponding to $\left\langle\mathbf{S}_{i}^{z}\right\rangle=1 / 2$, or the spin full polarization. We have calculated the critical field $\mu B_{c}$ (defined as the lowest field to induce the full spin polarization) as a function of $J^{\prime} / J$. The results are plotted in Fig. 3. As we can see, $\mu B_{c}$ increases as $J^{\prime}$ increases for the fixed $J$. As $J^{\prime}$ increases, the spin couplings are strengthened, and it requires a higher field to polarize the spin.

Very recently, Coldea et al. ${ }^{10}$ reported an neutron scattering experiments on the antiferromagnet $\mathrm{Cs}_{2} \mathrm{CuCl}_{4}$ in the high magnetic field. That system is a quasi-2D spin-1/2 quantum system in a triangular lattice, as shown in Fig. 1. The spinspin couplings are anisotropic with $J^{\prime} / J \approx 0.33$. In the absence of an external magnetic field, the spins are incommensurately ordered and are aligned within the plane of the triangular lattice. The latter may indicate a weak deviation from the Heisenberg model. Coldea et al. studied the low temperature states of the system in the presence of in-plane as well as perpendicular magnetic fields. In the presence of perpendicular fields, the states are found to be magnetically ordered with a varying incommensuration below a critical field, above which the system becomes a fully spin polarized ferromagnet. In the presence of an in-plane field, they have observed additional spin liquid phase between the incommensurate states and the ferromagnetic phase. There have been theoretical efforts to understand their experimental results. ${ }^{16}$ In the present paper, we have only considered the Heisenberg model in a uniform magnetic field. The predicted spin structure breaks the SU(2) symmetry, and shows the spin polarization along the field direction. Such a spin structure is compatible with the experiments in a perpendicular field, but incompatible with the in-plane field. Therefore, our MFT may be of relevance to the perpendicular field case in their experiments.

To compare with the experiments, we define a quantity to describe the incommensuration, which is proportional to the 
TABLE I. The incommensuration to the Néel state along the $b$ axis is listed. $h^{*}=0$ means the absence the magnetic field, and $h^{*}=1$ means the state is saturated fully. SE means the series expansion method, and the data are estimated from the work of Ref. 3, which was also predicted by Manuel and Ceccatto (Ref. 5).

\begin{tabular}{lcccc}
\hline \hline$h^{*}$ & Exp. (Ref. 10) & $J^{\prime} / J=0.404$ & $J^{\prime} / J=0.33$ & SE (Ref. 3) \\
\hline $0.0\left(\epsilon_{0}\right)$ & 0.03 & 0.047 & 0.035 & 0.028 \\
\hline $1.0\left(\epsilon_{c}\right)$ & 0.053 & 0.053 & 0.0422 & - \\
\hline \hline
\end{tabular}

wave vector deviation from the Néel state, $\epsilon=1 / 2-2 k_{0}^{*} / \pi$. In Table I, we list the experimental ${ }^{10}$ and theoretical values of $\epsilon$ at field $h^{*}=0$ and at the critical field $h^{*}=1$ for the two values of $J^{\prime} / J$. Also listed are the values of $\epsilon$ at $h^{*}=0$ calculated from the series expansion method. ${ }^{3}$ The agreement between the present MFT and the series expansion at $h^{*}$ $=0$ is very good. In Fig. 4, we plot the incommensuration relative to the Néel state as a function of the external field for $J^{\prime} / J=0.33$. Our mean field results are in qualitative agreement with the experiments: as the field increases, $\epsilon$ also increases in the parameter space of interest. Quantitatively, the theory predicts a weaker variation in the incommensuration than in the experiments. This discrepancy could be partly due to the neglect of the deviation of the physical system from the Heisenberg model in the theory.

In summary, we have used a Schwinger boson mean field theory to study the antiferromagnetic Heisenberg model on an anisotropic triangular lattice in the presence of an external magnetic field. We calculate the magnetic field dependence

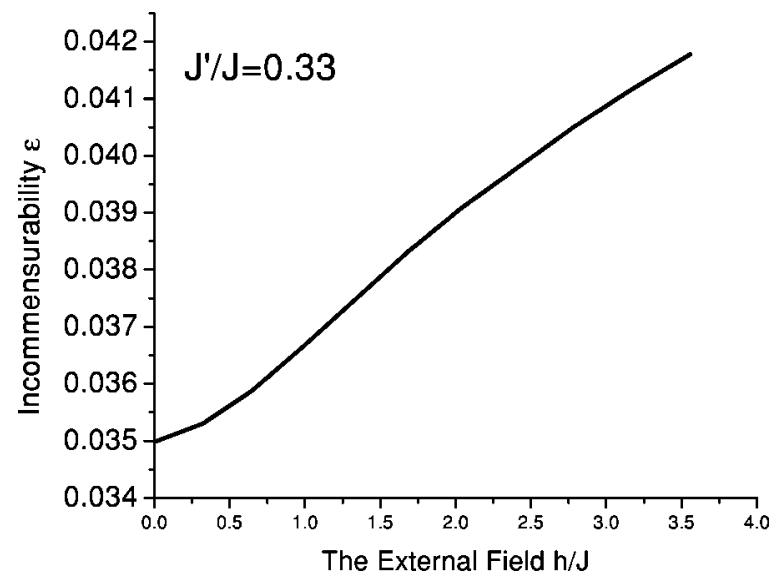

FIG. 4. The incommensuration $\epsilon$ vs the external field at $J^{\prime} / J$ $=0.33$.

of the incommensurability of the spin spiral phase. The theoretical results are compared well with the recent neutron scattering experiments.

We would like to acknowledge stimulating discussions with D. A. Tennant and R. Coldea on their experiments. This work was supported by a grant from the Research Grants Council of Hong Kong (Project HKU7109/02P) and a CRCG grant of the University of Hong Kong, by the U.S. DOE Grant No. FG03-01ER45687, and by the Chinese Academy of Sciences. The authors also acknowledge ICTP at Trieste, Italy for its support and hospitality, where part of the work was initiated.
${ }^{1}$ S. Chakravarty, B.I. Halperin, and D.R. Nelson, Phys. Rev. B 39, 2344 (1989).

${ }^{2}$ A. Auerbach, Interacting Electrons and Quantum Magnetism (Springer-Verlag, New York, 1994).

${ }^{3}$ Zheng Weihong, R.H. McKenzie, and R.R.P. Singh, Phys. Rev. B 59, 14367 (1999).

${ }^{4}$ J. Merino, R.H. McKenzie, J.B. Marston, and C.H. Chung, J. Phys.: Condens. Matter 11, 2965 (1999).

${ }^{5}$ L.O. Manuel and H.A. Ceccatto, Phys. Rev. B 60, 9489 (1999).

${ }^{6}$ C.H. Chung, J.B. Marston, and R.H. Mckenzie, J. Phys.: Condens. Matter 13, 5159 (2001).

${ }^{7}$ R.H. McKenzie, Comments Condens. Matter Phys. 18, 309 (1998).

${ }^{8}$ J. Schmalian, Phys. Rev. Lett. 81, 4232 (1998); H. Kino and H. Kontani, J. Phys. Soc. Jpn. 67, 3691 (1998); H. Kino and T. Moriya, ibid. 67, 3695 (1998); M. Votja and E. Dagotta, Phys. Rev. B 59, 713 (1999).
${ }^{9}$ R. Coldea, D.A. Tennant, R.A. Cowley, D.F. McMorrow, B. Dorner, and Z. Tylczynski, Phys. Rev. Lett. 79, 151 (1997).

${ }^{10}$ R. Coldea, D.A. Tennant, A.M. Tsvelik, and Z. Tylczynski, Phys. Rev. Lett. 86, 1335 (2001).

${ }^{11}$ A. Auerbach and D.P. Arovas, Phys. Rev. Lett. 61, 617 (1988); D.P. Arovas and A. Auerbach, Phys. Rev. B 38, 316 (1988).

${ }^{12}$ I. Richey and P. Coleman, J. Phys.: Condens. Matter 2, 9227 (1990); D. Yoshioka and J. Miyazaki, J. Phys. Soc. Jpn. 60, 614 (1991); A. Mattsson, Phys. Rev. B 51, 11574 (1995).

${ }^{13}$ D. Yoshioka, J. Phys. Soc. Jpn. 58, 32 (1989); S. Sarker, C. Jayaprakash, H.R. Krishnamurthy, and M. Ma, Phys. Rev. B 40, 5028 (1989).

${ }^{14}$ H. Bethe, Z. Phys. 71, 205 (1931).

${ }^{15}$ S. Q. Shen, Int. J. Mod. Phys. B 12, 709 (1998).

${ }^{16}$ M. Bocquet, F.H.L. Essler, A. M. Tsvelik, and A. O. Gogolin, Phys. Rev. B 64, 094425 (2001). 